\title{
Extended Target Tracking Using Polynomials With Applications to Road-Map Estimation
}

\author{
Christian Lundquist, Umut Orguner, Member, IEEE, and Fredrik Gustafsson, Senior Member, IEEE
}

\begin{abstract}
This paper presents an extended target tracking framework which uses polynomials in order to model extended objects in the scene of interest from imagery sensor data. State space models are proposed for the extended objects which enables the use of Kalman filters in tracking. Different methodologies of designing measurement equations are investigated. A general target tracking algorithm that utilizes a specific data association method for the extended targets is presented. The overall algorithm must always use some form of prior information in order to detect and initialize extended tracks from the point tracks in the scene. This aspect of the problem is illustrated on a real life example of road-map estimation from automotive radar reports along with the results of the study.
\end{abstract}

Index Terms-Extended target tracking, polynomial, parabola, errors in output, errors in variables, EIV, data association, automotive radar, road map.

\section{INTRODUCTION}

$\mathbf{T}$ HIS contribution relates to tracking applications where a sensor platform observes objects in local coordinates $(\mathrm{x}, \mathrm{y}, \mathrm{z})$. We focus on the case of a moving sensor platform and stationary objects, but the framework can be generalized to any target tracking scenario. Some of these detected objects are points, while others are extended objects. Tracking point shaped objects have been the main focus in the tracking literature for a long time [1], [2]. In particular in the computer vision literature, tracking extended objects has become popular during the last decades. The literature models extended objects as closed contours, like simple geometrical shapes as rectangles and ellipses, or arbitrary shapes as splines [3] and active contours [4]. We propose to complement these models of extended objects with detection and data association methods for sensors providing point measurements. Letting $\times$ be the longitudinal, $y$ the lateral and $z$ the vertical direction, possible applications include the following three automotive and two naval examples:

- Road curvature models $\mathrm{y}=f(\mathrm{x} ; \theta)$ based on lane markers detected by a vision sensor. Such models are needed in all future collision avoidance systems, in particular lane assist systems [5].

- Similar extended objects along the road side $\mathrm{y}=f(\mathrm{x} ; \theta)$ based on radar mainly. Such a model is a useful complement to the vision based road model above, or for au-

Copyright (c) 2010 IEEE. Personal use of this material is permitted. However, permission to use this material for any other purposes must be obtained from the IEEE by sending a request to pubs-permissions@iee.org.

The authors are with the Department of Electrical Engineering, Division of Automatic Control, Linköping University, Linköping, SE-581 83, Sweden, e-mail: \{lundquist,umut,fredrik\}@isy.liu.se. tonomous vehicles driving in unstructured environments. This is our application example in Section VI.

- Road inclination models $\mathrm{z}=f(\mathrm{x} ; \theta)$ based on a combination of vision and radar with good azimuth resolution. This can be used in look-ahead control [6] for fuel efficient cruise controllers.

- Shore models $\mathrm{x}=f(\mathrm{y} ; \theta)$ based on a radar to describe the shape of islands and parts of the coastline. This can be used in robust navigation systems based on map matching, see for instance the extended approach in [7], [8].

- Vertical shape models $\mathrm{z}=f(\mathrm{y} ; \theta)$ based on a camera describing the altitude variations, for instance, of an island as seen from the sensor platform. Applications are similar to the previous point.

The common theme in these examples is that the model is parameterized in a given model class (we will study polynomials), and that both the input and output are observed variables, leading to an errors in variables (EIV) [9] problem. This is, to the best of the authors knowledge, an original approach to describe extended objects. We derive a modified measurement update for the EIV model, and demonstrate its increased accuracy compared to the straightforward errors in output (EIO) approximation for filtering applications. The detection procedure requires some form of prior information on the shape of the extended object because many different models can suit the same set of measurements.

This paper is outlined as follows. We begin with an overview of the related literature in Section II. Connections to several different fields of research are pointed out and this makes this section fairly long. However, the subsequent sections are organized such that the uninterested reader can skip this section without causing a loss of understanding for the the rest of the paper. A basic problem formulation of point and extended target tracking using polynomials as extended objects is given in Section III. The state space representations that we are going to use in extended target tracking are introduced in Section IV. Our general algorithm for tracking point and extended targets is summarized in Section V. Section VI introduces the practical application. We apply the ideas presented in earlier sections to the road map estimation problem utilizing the automotive radar reports. The experiments done and the results obtained are presented in Section VI-C. The paper is concluded in Section VII.

\section{RELATED Literature}

Extended target tracking has been studied in the literature for some years and we give a brief overview in Section II-A. 
Section II-B summarizes a couple of estimation methods proposed earlier for curves and contours. One of our proposed solutions is stated to be related to an estimation framework called as errors in variables problem and some references for this are given in Section II-C. Finally, different solutions related to our motivating application example of road edge estimation are discussed in Section II-D.

\section{A. Extended Target Tracking}

A target is denoted extended whenever the target extent is larger than the sensor resolution, i.e., it is large enough to give rise to more than one measurement per time step. Common methods used to track extended objects are very similar to the ones used for tracking a group of targets moving in formation, see e.g., [10]. The bibliography [11] provides a comprehensive overview of existing literature in the area of group and cluster tracking. One conventional method is to model the target as a set of point features in a target reference frame, each of which may contribute at most one sensor measurement [12]. The exact location of a feature in the target reference frame is often assumed uncertain. The motion of an extended target is modeled through the process model in terms of the translation and rotation of the target reference frame relative to a world coordinate frame, see e.g., [13]. As is the case most of the time, not all measurements arise from features belonging to targets and some are due to false detections (clutter). The association hypotheses are derived through some data association algorithm. In [14] a method is proposed where the association hypotheses are included in the state vector and the output of the tracking filter is a joint posterior density function of the state vector and the association hypotheses.

Instead of modeling the target as a number of point features, the target may be represented by a spatial probability distribution. It is more likely that a measurement comes from a region of high spatial density than from a sparse region. In [15], [16] it is assumed that the number of received target and clutter measurements are Poisson distributed, hence several measurements may originate from the same target. Each target related measurement is an independent sample from the spatial distribution. The spatial distribution is preferable where the point source models are poor representations of reality, that is, in the cases where the measurement generation is diffuse. In [17] a similar approach is presented, but since raw data is considered, no data association hypotheses are needed.

In many papers dealing with the shape of a target it is assumed that the sensor is also able to measure one or more dimensions of the target's extent. A high-resolution radar sensor may provide measurements of a targets down-range extent, i.e., the extension of the objects along the line-of-sight. The information of the target's extent is incorporated in the tracking filter aiding the tracking process to maintain track on the target when it is close to other objects. An elliptical target model, to represent an extended target or a group of targets, is proposed in [18]. The idea is improved in [19] with a solution based on EKF, in [20] based on UKF and in [21] with a solution based on a Monte Carlo algorithm.

\section{B. Contours and Curves}

Modeling of extended targets in this work is very similar to active contours [4] and snakes [22], which model the outline of an object based on 2 dimensional image data, studied extensively in computer vision. The active contour models are also polynomials represented by B-splines. Moreover, the probabilistic processing of active contours from image data pioneered mainly by Blake and Isard [4] uses similar estimation framework like Kalman or particle filters. The underlying low-level processing involved, on the other hand, assumes reasonably the existence of image data from which features (like Harris corners) or feature maps (like Harris measures etc.) can be extracted. The so-called Condensation algorithm [23], for example, searches for suitable sets of features in the image data (or in the feature map) iteratively for each of its different hypotheses (particles) in the calculation of the likelihoods. In this respect, the active contour framework would be an important candidate for doing tracking with the raw sensor data without any thresholding which is named as "trackbefore-detect" in the target tracking literature. The approach presented in this work carries the distinction of working only with a single set of features provided most of the time by thresholding of the raw sensor data (like conventional target tracking) and hence is mainly aimed at applications where the users (of the sensors) either are unable to reach or do not have the computation power to work with the raw sensor data.

The mapping of the boundaries of complex objects is also achieved in [24] using splinegon techniques and a range sensor such as e.g., laser mounted on moving vehicles.

\section{Errors In Variables}

The use of "noisy" measurements as model parameters in this work makes this paper directly related to the errors in variables framework, where some of the independent variables are contaminated by noise. Such a case is common in the field of system identification [25] when not only the system outputs, but also the inputs are measured imperfectly. Examples of such EIV representations can be found in [9] and a representative solution is proposed in [26], [27]. The Kalman filter cannot be directly applied to such EIV processes as discussed in [26], [28]. Nevertheless, an extension of the Kalman filter, where the state and the output are optimally estimated in the presence of state and output noise is proposed in [29]. The EIV problem is also closely related to the total least squares methodology, which is well described in the papers [30]-[32], in the survey paper [33] and in the textbook [34].

\section{Application}

An important part of this contribution is the application of the ideas to the real world example of road map estimation using automotive radar sensor reports. In this context, the point and extended targets existing in the scene would represent e.g., lamp posts and guardrails along the road, respectively. Our earlier work [35] contains the findings from similar tracking scenarios. This also connects our work to the approaches presented in the literature for the problem of road edge 
estimation. The third order approximation of the two sided (left and right) "clothoid model" has been used in connection with Kalman filters in [36] and [37] for laser scanner measurements and radar measurements, respectively. In [38] two road edge models are proposed, one of which is very similar to the model proposed in [37], and used a constrained quadratic program to solve for the parameters. A linear model represented by its midpoint and orientation (one for each side of the road) is utilized by [39] with lidar sensing for tracking road-curbs. Later, the results of [39] were enhanced in [40] with the addition of image sensors. A similar extended Kalman filtering based solution is given in [41], where a circular road edge modeling framework is used. Recently, the particle filters (also referred to as condensation in image and video processing) have been applied to the road edge estimation problem in [42] with an hyperbolic road model.

\section{PRoblem Formulation}

The examples mentioned in Section I all involve 2dimensional extended objects, modeled by any configuration of two local coordinates ( $x, y$ or $z$ ). In the following parts of the paper, we describe all models in the $x-y$-plane without any loss of generality, i.e., the coordinates are easily exchanged to describe objects in any other space.

Suppose we are given the sensor measurements in batches of Cartesian $\mathrm{x}$ and $\mathrm{y}$ coordinates as follows:

$$
\left\{\mathbf{z}_{k}^{(i)} \triangleq\left[\mathrm{x}^{(i)} \quad \mathrm{y}^{(i)}\right]_{k}^{\mathrm{T}}\right\}_{i=1}^{N_{z_{k}}}
$$

for discrete time instants $k=1, \ldots, K$. In many cases in reality (e.g., radar, laser and stereo vision) and in the practical application considered in this work, the sensor provides range $r$ and azimuth angle $\psi$ given as

$$
\left\{\overline{\mathbf{z}}_{k}^{(i)} \triangleq\left[\mathrm{r}^{(i)} \quad \psi^{(i)}\right]_{k}^{\mathrm{T}}\right\}_{i=1}^{N_{z_{k}}}
$$

In such a case we assume that some suitable standard polar to Cartesian conversion algorithm is used to convert these measurements into the form (1).

The measurements $\mathbf{z}$ are noisy point measurements originating from one of the following sources

- Point sources with state vector

$$
\mathbf{x}_{\mathrm{P}} \triangleq\left[\begin{array}{ll}
\mathrm{x} & \mathrm{y}
\end{array}\right]^{\mathrm{T}}
$$

which represents the true Cartesian position of the source.

- Extended sources which are represented by an $n^{\text {th }}$ order polynomial given as

$$
\mathrm{y}=a_{0}+a_{1} \mathrm{x}+a_{2} \mathrm{x}^{2}+\ldots+a_{n} \mathrm{x}^{n}
$$

in the range $\left[\mathrm{x}_{\text {start }}, \mathrm{x}_{\text {end }}\right]$ where $\mathrm{x}_{a} \triangleq\left[\begin{array}{llll}a_{0} & a_{1} & \cdots & a_{n}\end{array}\right]^{\mathrm{T}}$ are the polynomial coefficients and $\left[\begin{array}{ll}x & y\end{array}\right]^{\mathrm{T}}$ are planar Cartesian coordinates. Note that the coordinate $y$ is a function of $x$ and that the direction of the coordinate frame is chosen dependent on the application in mind. The state vector is defined as

$$
\mathbf{x}_{\mathrm{L}} \triangleq\left[\begin{array}{lll}
\mathbf{x}_{a} & \mathrm{x}_{\text {start }} & \mathrm{x}_{\mathrm{end}}
\end{array}\right]^{\mathrm{T}} .
$$

- False detections
The state models considered in this contribution are described, in general, by the state space equations

$$
\begin{aligned}
\mathbf{x}_{k+1} & =f\left(\mathbf{x}_{k}, \mathbf{u}_{k}\right)+\mathbf{w}_{k}, \\
\mathbf{y}_{k} & =h\left(\mathbf{x}_{k}, \mathbf{u}_{k}\right)+\mathbf{e}_{k},
\end{aligned}
$$

where $\mathbf{x}, \mathbf{u}$ and $\mathbf{y}$ denotes the state, the input signal, and the output signal, while $\mathbf{w} \sim \mathcal{N}(0, Q)$ and $\mathbf{e} \sim \mathcal{N}(0, R)$ are the process and measurement noise, respectively. The use of an input signal is explained in Section IV-A. The time index is denoted with $k$.

One of the main contributions of our work is the specific state space representation we propose for extended targets which is presented in Section IV. A polynomial is generally difficult to handle in a filter, since the noisy measurements are distributed arbitrarily along the polynomial. In this respect, the measurement model (6b) contains parts of the actual measurement vector as parameters. For the sake of simplicity, the tracked objects are assumed stationary, resulting in very simple motion models (6a). However, the motion or process model may easily be substituted and chosen arbitrarily to best fit its purpose.

The aim is to obtain posterior estimates of the point sources $\left\{\mathbf{x}_{\mathrm{P}, k \mid k}^{(i)}\right\}_{i=1}^{N_{\mathrm{P}}}$ and the extended sources $\left\{\mathbf{x}_{\mathrm{L}, k \mid k}^{(i)}\right\}_{i=1}^{N_{\mathrm{L}}}$ given all the measurements $\left\{\left\{\mathbf{z}_{\ell}^{(i)}\right\}_{i=1}^{N_{z_{\ell}}}\right\}_{\ell=1}^{k}$ recursively in time.

\section{State Space Representation for an Extended OBJECT}

This section first investigates the measurement model to be used with extended sources in Section IV-A and then makes short remarks about the state dynamics equation for polynomials in Section IV-B.

\section{A. Measurement Model}

This section describes how a point measurement $\mathbf{z}$ relates to the state $\mathrm{x}_{\mathrm{L}}$ of an extended object. For this purpose, we derive a measurement model in the form (6b), which describes the relation between the state variables $\mathrm{x}_{\mathrm{L}}$, defined in (5), and output signals $\mathbf{y}$ and input signals $\mathbf{u}$. Notice that, for the sake of simplicity, we also drop the subscripts $k$ specifying the time stamps of the quantities.

The general convention in modeling is to make the definitions

$$
\mathbf{y} \triangleq \mathbf{z}, \quad \mathbf{u} \triangleq \emptyset,
$$

where $\emptyset$ denotes the empty set meaning that there is no input. In this setting, it is extremely difficult, if not impossible, to find a measurement model connecting the outputs $y$ to the states $\mathrm{x}_{\mathrm{L}}$ in the form of (6b). Therefore, we are forced to use other selections for $\mathbf{y}$ and $\mathbf{u}$. Here, we make the selection

$$
\mathbf{y} \triangleq \mathrm{y}, \quad \mathbf{u} \triangleq \mathrm{x} .
$$

Although being quite a simple selection, this choice results in a rather convenient linear measurement model in the state partition $\mathbf{x}_{a}$,

$$
\mathbf{y}=H_{a}(\mathbf{u}) \mathbf{x}_{a}+\mathbf{e}_{a}
$$


where $H_{a}(\mathbf{u})=\left[\begin{array}{lllll}1 & \mathrm{x} & \mathrm{x}^{2} & \cdots & \mathrm{x}^{n}\end{array}\right]^{\mathrm{T}}$. It is the selection in (8) rather than (7) that allows us to use the standard methods in target tracking with clever modifications. Such a selection as (8) is also in accordance with the errors in variables representations where measurement noise are present in both the outputs and inputs, i.e., the observation $\mathbf{z}$ can be partitioned according to

$$
\mathbf{z}=\left[\begin{array}{l}
\mathbf{u} \\
\mathbf{y}
\end{array}\right]
$$

We express the measurement vector given in (1) in terms of a noise free variable $\mathbf{z}_{0}$ which is corrupted by additive measurement noise $\tilde{\mathbf{z}}$ according to

$$
\mathbf{z}=\mathbf{z}_{0}+\tilde{\mathbf{z}}, \quad \tilde{\mathbf{z}} \sim \mathcal{N}\left(0, \Sigma_{\mathrm{c}}\right)
$$

where the covariance $\Sigma_{\mathrm{c}}$ can be decomposed as

$$
\Sigma_{\mathrm{c}}=\left[\begin{array}{cc}
\Sigma_{\mathrm{x}} & \Sigma_{\mathrm{xy}} \\
\Sigma_{\mathrm{xy}} & \Sigma_{\mathrm{y}}
\end{array}\right] .
$$

Note that, in the case the sensor provides measurements only in polar coordinates (2), one has to convert both the measurement $\overline{\mathbf{z}}$ and the measurement noise covariance

$$
\Sigma_{\mathrm{p}}=\operatorname{diag}\left(\sigma_{\mathrm{r}}^{2}, \sigma_{\psi}^{2}\right)
$$

into Cartesian coordinates, which is a rather standard procedure. Note that, in such a case, the resulting Cartesian measurement covariance $\Sigma_{c}$ is, in general, not necessarily diagonal and hence $\Sigma_{x y}$ of (12) might be non-zero.

Since the model (9) is conditionally linear given the measurements, the Kalman filter measurement update formulas can be used to incorporate the information in $\mathbf{z}$ into the extended source state $\mathrm{x}_{\mathrm{L}}$. An important question in this regard is what would be the measurement covariance of the measurement noise term $\mathbf{e}_{a}$ in (9). This problem can be tackled in two ways.

1) Errors in Output (EIO) scheme: Although the input terms defined in (8) are measured quantities (and hence affected by the measurement noise), and therefore the model parameters $H_{a}(\mathbf{u})$ are uncertain, in a range of practical applications where parameters are obtained from measurements, such errors are neglected. Thus, the first scheme we present here neglects all the errors in $H_{a}(\mathbf{u})$. In this case, it can easily be seen that

$$
\mathbf{e}_{a}=\tilde{\mathbf{y}}
$$

and therefore the covariance $\Sigma_{a}$ of $\mathbf{e}_{a}$ is

$$
\Sigma_{a}=\Sigma_{\mathrm{y}}
$$

This type of approach was also used in our previous work [35] which presented earlier versions of the findings in this paper.

2) Errors in Variables (EIV) scheme: Neglecting the errors in the model parameters $H_{a}(\mathbf{u})$ can cause overconfidence in the estimates of recursive filters and can actually make data association difficult in tracking applications (by causing too small gates). We, in this second scheme, use a simple methodology to take the uncertainties in $H_{a}(\mathbf{u})$ into account in line with EIV framework. Assuming that the elements of the noise free quantity $\mathbf{z}_{0}$ satisfy the polynomial equation exactly, we get

$$
\begin{aligned}
& \mathbf{y}-\tilde{\mathbf{y}}=H_{a}(\mathbf{u}-\tilde{\mathbf{u}}) \mathbf{x}_{a}, \\
& \mathrm{y}-\tilde{\mathrm{y}}=\left[\begin{array}{lllll}
1 & \mathrm{x}-\tilde{\mathrm{x}} & (\mathrm{x}-\tilde{\mathrm{x}})^{2} & \cdots & (\mathrm{x}-\tilde{\mathrm{x}})^{n}
\end{array}\right] \mathbf{x}_{a},
\end{aligned}
$$

which can be approximated using a first order Taylor expansion resulting in

$$
\begin{aligned}
\mathbf{y} & \approx H_{a}(\mathbf{u}) \mathbf{x}_{a}-\tilde{H}_{a}(\mathbf{u}) \tilde{\mathbf{x}} \mathbf{x}_{a}+\tilde{\mathrm{y}} \\
& =H_{a}(\mathbf{u}) \mathbf{x}_{a}+\tilde{h}_{a}\left(\mathbf{x}_{a}, \mathbf{u}\right)\left[\begin{array}{c}
\tilde{\mathrm{x}} \\
\tilde{y}
\end{array}\right]
\end{aligned}
$$

with

$$
\begin{aligned}
H_{a}(\mathbf{u}) & =\left[\begin{array}{lllll}
1 & \mathrm{x} & \mathrm{x}^{2} & \cdots & \mathrm{x}^{n}
\end{array}\right], \\
\widetilde{H}_{a}(\mathbf{u}) & =\left[\begin{array}{lllll}
0 & 1 & 2 \mathrm{x} & \cdots & n \mathrm{x}^{n-1}
\end{array}\right], \\
\tilde{h}_{a}\left(\mathbf{x}_{a}, \mathbf{u}\right) & =\left[\begin{array}{llll}
-a_{1}-2 a_{2} \mathrm{x}-\cdots-n a_{n} \mathrm{x}^{n-1} & 1
\end{array}\right] .
\end{aligned}
$$

Hence, the noise term $\mathbf{e}_{a}$ of (9) is given by

$$
\mathbf{e}_{a}=\tilde{\mathrm{y}}-\tilde{H}_{a} \tilde{\mathrm{x}} \mathbf{x}_{a}=\tilde{h}_{a}\left(\mathbf{x}_{a}, \mathbf{u}\right)\left[\begin{array}{l}
\tilde{\mathrm{x}} \\
\tilde{\mathrm{y}}
\end{array}\right]
$$

and its covariance is given by

$$
\begin{aligned}
\Sigma_{a}=\mathrm{E}\left(\mathbf{e}_{a} \mathbf{e}_{a}^{\mathrm{T}}\right) & =\Sigma_{\mathrm{y}}+\widetilde{H}_{a} \mathbf{x}_{a} \Sigma_{\mathbf{x}} \mathbf{x}_{a}^{\mathrm{T}} \widetilde{H}_{a}^{\mathrm{T}}-2 \widetilde{H}_{a} \mathbf{x}_{a} \Sigma_{\mathrm{xy}} \\
& =\tilde{h}_{a}\left(\mathbf{x}_{a}, \mathbf{u}\right) \Sigma_{\mathrm{c}} \tilde{h}_{a}^{\mathrm{T}}\left(\mathbf{x}_{a}, \mathbf{u}\right) .
\end{aligned}
$$

Note that the EIV covariance $\Sigma_{a}$ depends on the state variable $\mathbf{x}_{a}$.

3) Example: An example is used to compare the performances of the EIO and the EIV schemes. A second order polynomial with the true states

$$
\mathbf{x}_{a}=\left[\begin{array}{lll}
a_{0} & a_{1} & a_{2}
\end{array}\right]^{\mathrm{T}}=\left[\begin{array}{lll}
-20 & -0.5 & 0.008
\end{array}\right]^{\mathrm{T}}
$$

is used and 100 uniformly distributed measurements are extracted in the range $x=[0,200]$. The measurements are given on polar form as in (2) and zero mean Gaussian measurement noise with covariance as in (13) is added using the parameters

$$
\begin{array}{llll}
\text { Sensor 1: } & \sigma_{\mathrm{r} 1}=0.5, & \sigma_{\psi 1}=0.05, \\
\text { Sensor 2: } & \sigma_{\mathrm{r} 2}=10, & \sigma_{\psi 2}=0.05, \\
\text { Sensor 3: } & \sigma_{\mathrm{r} 3}=10, & \sigma_{\psi 3}=0.005,
\end{array}
$$

to simulate different type of sensors. Sensor 1 represents a sensor with better range than bearing accuracy, whereas the opposite holds for sensor 3. Sensor 2 has about the same range and bearing accuracies. The true polynomial and the measurements are shown in Fig. 1. The measurements are transformed into Cartesian coordinates. The following batch methods $\left(N_{z_{k}}=100, K=1\right)$ are applied to estimate the states

- Least squares (LS EIO) estimator,

- Weighted least squares (WLS EIO) with EIO covariance,

- Weighted least squares (WLS EIV) with EIV covariance. The state $\mathbf{x}_{a}$ used in (19) is estimated through a least squares solution in advance.

Furthermore, the states are estimated recursively $\left(N_{z_{k}}=1\right.$, $K=100)$ using

- Kalman filter (KF EIO) with EIO covariance, 


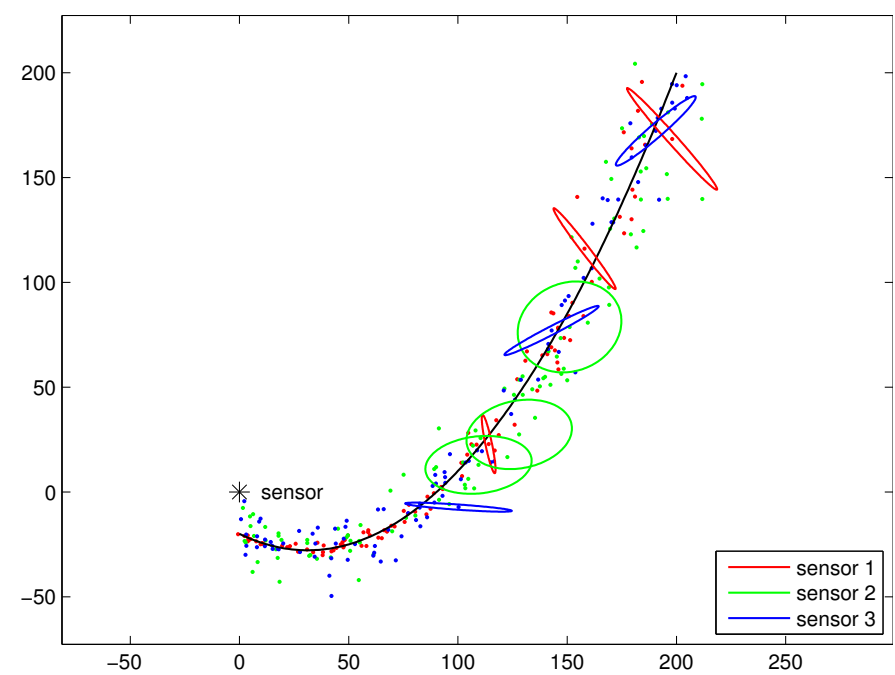

Fig. 1. The true polynomial and the extracted measurements are shown. Covariance ellipses are drawn for a few measurements to show the direction of the uncertainties.

- Kalman filter (KF EIV) with EIV covariance. The predicted state estimate $\hat{\mathbf{x}}_{a, k \mid k-1}$ is used in (19) to derive $R_{a, k}$.

- Unscented Kalman filter (UKF EIV) with sigma points derived by augmenting the state vector with the noise terms

$$
\tilde{\mathbf{z}}=\left[\begin{array}{ll}
\tilde{\mathbf{u}} & \tilde{\mathbf{y}}
\end{array}\right]^{\mathrm{T}}, \quad \tilde{\mathbf{z}} \sim \mathcal{N}\left(0, \Sigma_{\mathrm{c}}\right),
$$

to consider the error in all directions (as in the EIV case), and to deal with the nonlinear transformations of the noise terms.

The covariance of the process noise is set to zero, i.e., $Q=0$, since the target is not moving. The initial conditions of the state estimate are selected as

$$
\begin{aligned}
& \hat{\mathbf{x}}_{a, 0}=\left[\begin{array}{lll}
0 & 0 & 0
\end{array}\right]^{\mathrm{T}}, \\
& P_{a, 0}=\left(\frac{\sigma_{a}^{2}}{\kappa} \operatorname{diag}\left(\mathbf{x}_{a}\right)\right)^{2},
\end{aligned}
$$

where $\kappa=3$ and $\sigma_{a}^{2}=8$. Note that every estimate's initial uncertainty is set to be a scaled version of its true value in (23b).

The RMSE values for 1000 Monte Carlo simulations are given in Table I. The RMSE of the EIV schemes is clearly lower than the other methods, especially for sensor 1 and 3 with non-symmetric measurement noise. This result justifies the extra computations required for calculating the EIV covariance. There is only a small difference in the performance between the KF EIV and the UKF EIV for the second order polynomial. This is a clear indication of that the simple Taylor series approximation used in deriving the EIV covariance is accurate enough.

Remark 1 (Robustness): We use only Gaussian noise representations in this paper which would directly connect our work to the (implicit) minimization of LS-type cost functions which are known to be non-robust against outliers. The algorithms we are going to propose for estimation are going to be protected, to some extent, against this by classical techniques like gating
TABLE I

RMSE VALUES FOR THE EXTENDED OBJECT IN FIGURE 1. THE SENSOR CONFIGURATIONS ARE DEFINED IN (21)

\begin{tabular}{ll|lll|lll}
\hline \multicolumn{2}{l}{ Sensor } & LS & WLS & WLS & KF & KF & UKF \\
& & EIO & EIO & EIV & EIO & EIV & EIV \\
\hline \multirow{3}{*}{1} & $a_{0}$ & 5.10 & 0.55 & 0.45 & 0.55 & 0.48 & 0.49 \\
& $a_{1}$ & 0.18 & 0.034 & 0.024 & 0.034 & 0.029 & 0.029 \\
& $a_{2} \cdot 10^{-3}$ & 1.06 & 0.29 & 0.24 & 0.29 & 0.31 & 0.32 \\
\hline \multirow{2}{*}{2} & $a_{0}$ & 4.90 & 3.54 & 3.35 & 2.90 & 2.44 & 2.36 \\
& $a_{1}$ & 0.20 & 0.11 & 0.099 & 0.10 & 0.11 & 0.10 \\
& $a_{2} \cdot 10^{-3}$ & 1.21 & 0.77 & 0.66 & 0.78 & 0.83 & 0.80 \\
\hline \multirow{3}{*}{3} & $a_{0}$ & 2.53 & 30.51 & 3.51 & 30.47 & 4.81 & 4.35 \\
& $a_{1}$ & 0.068 & 0.45 & 0.072 & 0.45 & 0.12 & 0.12 \\
& $a_{2} \cdot 10^{-3}$ & 0.39 & 1.27 & 0.40 & 1.26 & 0.62 & 0.60 \\
\hline \multicolumn{7}{l}{}
\end{tabular}

[1], [2]. Extra robustness features can be accommodated by the use of Huber [43] and $\ell_{1}$ norms etc. in the estimation which were considered to be outside the scope of this paper.

4) Complete Measurement Model for an Extended Object: Up to this point, we have only considered how the observation $\mathbf{z}$ relates to the state component $\mathbf{x}_{a}$. It remains to discuss the relation between the observation and the start $x_{\text {start }}$ and the end points $x_{e n d}$ of the polynomial. The measurement information must only be used to update these components of the state if the new observations of the extended source lie outside the range of the polynomial. We can define the following (measurement dependent) measurement matrix for this purpose:

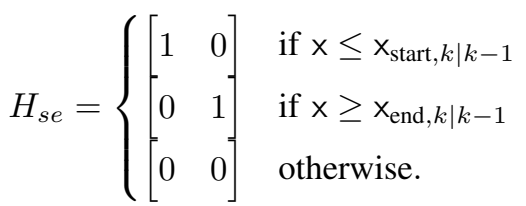

The complete measurement model of an extended object can now be summarized by

$$
\mathbf{z}=H_{\mathrm{L}} \mathbf{x}_{\mathrm{L}}+\mathbf{e}, \quad \mathbf{e} \sim \mathcal{N}\left(0, R_{\mathrm{L}}\left(\mathbf{x}_{\mathrm{L}}\right)\right),
$$

with

$$
\begin{aligned}
H_{\mathrm{L}} & =\left[\begin{array}{cc}
\mathbf{0}^{1 \times n} & H_{s e} \\
H_{a} & \mathbf{0}^{1 \times 2}
\end{array}\right] \\
R_{\mathrm{L}}\left(\mathbf{x}_{\mathrm{L}}\right) & =\operatorname{blkdiag}\left(\Sigma_{\mathrm{x}}, \Sigma_{a}\left(\mathbf{x}_{\mathrm{L}}\right)\right) .
\end{aligned}
$$

Put in words, if the $x$-component of a new measurement is closer to the sensor than the start point of the line $x_{\text {start }}$ it is considered in the measurement equation (24) and can used to update this state variable. Analogously, if a new measurement is more distant than the end point of the line $x_{\text {end }}$ it is considered in (24). Further, if a measurements is in between the start and end point of the line, the measurement model is zero in (24) and there is no relation between this measurement and the state variables $x_{\text {start }}$ or $x_{\text {end }}$.

\section{B. Process Model}

Any model as e.g., the standard constant velocity or coordinated turn model may be used for the targets. For simplicity it is assumed that the targets are stationary in this contribution, thus the process model on the form (6a) is linear and may be written

$$
\mathbf{x}_{k+1}=F \mathbf{x}_{k}+\mathbf{w}_{k}
$$


To increase the flexibility of the extended object an assumption about the dynamic behavior of its size is made. The size of the extended object is modeled to shrink with a factor $0.9<\lambda<1$ according to

$$
\begin{aligned}
\mathrm{x}_{\mathrm{start}, k+1} & =\mathrm{x}_{\mathrm{start}, k}+\lambda\left(\mathrm{x}_{\mathrm{end}, k}-\mathrm{x}_{\mathrm{start}, k}\right), \\
\mathrm{x}_{\mathrm{end}, k+1} & =\mathrm{x}_{\mathrm{end}, k}-\lambda\left(\mathrm{x}_{\mathrm{end}, k}-\mathrm{x}_{\mathrm{start}, k}\right),
\end{aligned}
$$

leading to the following process model for the polynomial

$$
F_{\mathrm{L}}=\left[\begin{array}{ccc}
\boldsymbol{I}^{n \times n} & \multicolumn{2}{c}{\mathbf{0}^{n \times 2}} \\
\mathbf{0}^{2 \times n} & 1-\lambda & \lambda \\
\lambda & 1-\lambda
\end{array}\right]
$$

This shrinking behavior for the polynomials allows for automatic adjustment of the start and end points of the polynomials according to the incoming measurements.

Some dynamics is included into the process model of the polynomials for the start and the end point components of the state, $x_{\text {start }}$ and $x_{\text {end }}$.

\section{Multi-Target Tracking Algorithm}

In this section, we describe the target tracking algorithm we use to track polynomial shaped extended objects and point objects at the same time. Suppose at time $k-1$, we have $N_{\mathrm{P}, k-1}$ estimated point objects whose states and covariances are given by $\left\{\hat{\mathbf{x}}_{\mathrm{P}, k-1 \mid k-1}^{(i)}, P_{\mathrm{P}, k-1 \mid k-1}^{(i)}\right\}_{i=1}^{N_{\mathrm{P}, k-1}}$ and $N_{\mathrm{L}, k-1}$ estimated extended objects whose states and covariances are given by $\left\{\hat{\mathbf{x}}_{\mathrm{L}, k-1 \mid k-1}^{(j)}, P_{\mathrm{L}, k-1 \mid k-1}^{(j)}\right\}_{j=1}^{N_{\mathrm{L}, k-1}}$. Basically, the task of the tracking algorithm is to obtain the updated states and the covariances $\left\{\hat{\mathbf{x}}_{\mathrm{P}, k \mid k}^{(i)}, P_{\mathrm{P}, k \mid k}^{(i)}\right\}_{i=1}^{N_{\mathrm{P}, k}},\left\{\hat{\mathbf{x}}_{\mathrm{L}, k \mid k}^{(j)}, P_{\mathrm{L}, k \mid k}^{(j)}\right\}_{j=1}^{N_{\mathrm{L}, k}}$ when the set of $N_{z_{k}}$ measurements given by $\left\{\mathbf{z}_{k}^{(m)}\right\}_{m=1}^{N_{z_{k}}}$ is obtained. Notice that the number of these updated summary statistics kept in the algorithm can change with appearance or disappearance of the objects in the environment. Hence, the number of each type of objects must also be estimated by the tracking algorithm.

We, in this work, consider a conventional multi-target tracking algorithm that uses a specific track initiation and deletion logic for handling the variable number of objects to track. Each cycle of estimation starts with the classical (association) hypotheses reduction technique "gating" which is followed by a data association process which determines the measurement to (point or extended) track associations. When the measurement to track associations are known, the current tracks are updated with their associated measurement. In this measurement update step, the results of Section IV-A are used for updating the extended object states and covariances. After the updates are completed one has to do track handling. This operation deals with

- Extended object generations from established point targets. This part of the tracking algorithm must depend heavily upon the problem under investigation and hence the prior information that one has about the possible extended objects because the mapping from a number of points to a number of polynomials is not unique.

- Track deletion. This operation deletes the old tracks that have not been associated to any measurements for a significant amount of time.
Below, in separate subsections, we investigate the steps of the tracking algorithm we propose in more detail.

\section{A. Gating and Data Association}

Each of the $N_{z_{k}}$ observations $\mathbf{z}_{k}^{(m)}, m=1, \ldots, N_{z_{k}}$ from the sensor measurements can be associated to one of the existing point tracks $\mathbf{x}_{\mathrm{P}}^{(i)}$, the extended tracks $\mathrm{x}_{\mathrm{L}}^{(j)}$ or a new point track is initiated. The number of association events (hypotheses) is extremely large. The classical technique to reduce the number of these hypotheses is called gating, see e.g., [44]. In this section we show how to apply gating and to make a nearest-neighbor type data association based on likelihood ratio tests. Other more complicated data association methods like multiple hypothesis tracking, according to e.g., [45], or joint probabilistic data association, as described by e.g., [44], can also be used in this framework with appropriate modifications for the extended sources. However, these are quite complicated and computationally costly approaches and the nearest neighbor type algorithm has been found to give sufficiently good performance for our application. The gating and the data association are performed according to the following calculations. The likelihood $\ell_{m, i}$ that the observation $\mathbf{z}^{(m)}$ corresponds to the $i^{\text {th }}$ point track $\mathrm{P}^{(i)}$ is given by

$$
\ell_{m, i}= \begin{cases}\mathcal{N}\left(\mathbf{z}_{k}^{(m)} ; \hat{\mathbf{z}}_{\mathrm{P}, k \mid k-1}^{(i)}, S_{\mathrm{P}, k \mid k-1}^{(i)}\right), & \text { if } \mathbf{z}_{k}^{(m)} \in \mathcal{G}_{\mathrm{P}}^{(i)} \\ 0, & \text { otherwise }\end{cases}
$$

where $\hat{\mathbf{z}}_{\mathrm{P}, k \mid k-1}^{(i)}$ is the predicted measurement of the point $\mathrm{P}_{i}$ according to the model (39) and $S_{\mathrm{P}, k \mid k-1}^{(i)}$ is its covariance (innovation covariance) in the Kalman filter. The gate $\mathcal{G}_{\mathrm{P}}^{(i)}$ is defined as the region

$$
\begin{aligned}
& \mathcal{G}_{\mathrm{P}}^{(i)} \triangleq\left\{\mathbf{z} \mid\left(\mathbf{z}-\hat{\mathbf{z}}_{\mathrm{P}, k \mid k-1}^{(i)}\right)^{\mathrm{T}}\left(S_{\mathrm{P}, k \mid k-1}^{(i)}\right)^{-1}\right. \\
& \left.\times\left(\mathbf{z}-\hat{\mathbf{z}}_{\mathrm{P}, k \mid k-1}^{(i)}\right) \leq \delta_{\mathrm{P}}\right\}
\end{aligned}
$$

where $\delta_{\mathrm{P}}$ is the gating threshold.

The likelihood $\ell_{m, j}$ that the observation $m$ belongs to the $j^{\text {th }}$ line is given by

$$
\ell_{m, j}= \begin{cases}\mathcal{N}\left(\mathbf{y}_{k}^{L,(m)} ; \hat{\mathbf{y}}_{\mathrm{L}, k \mid k-1}^{(j)}, S_{\mathrm{L}, k \mid k-1}^{(j)}\right), & \text { if } \mathbf{z}_{k}^{L,(m)} \in \mathcal{G}_{\mathrm{L}_{j}} \\ 0, & \text { otherwise }\end{cases}
$$

where $\hat{\mathbf{y}}_{\mathrm{L}, k \mid k-1}^{(j)}$ is the predicted output of the measurement model (9) and $S_{\mathrm{L}, k \mid k-1}^{(j)}$ is its covariance, both for the state estimates $\hat{\mathbf{x}}_{\mathrm{L}}^{(j)}$. The quantity $\mathbf{z}^{L,(m)}$ is representing the original measurement transformed into the line's corresponding coordinate frame and $\mathbf{y}_{k}^{L,(m)}$ is the $\mathrm{y}$ component of it. The gate $\mathcal{G}_{\mathrm{L}_{j}}$ is defined as

$$
\begin{aligned}
\mathcal{G}_{\mathrm{L}_{j}} \triangleq\{\mathbf{z}= & {\left[\begin{array}{l}
\mathbf{u} \\
\mathbf{y}
\end{array}\right] \mid \frac{\left(\mathbf{y}-\hat{\mathbf{y}}_{L, k \mid k-1}^{(j)}\right)^{2}}{S_{\mathrm{L}, k \mid k-1}^{(j)}} \leq \delta_{\mathrm{L}}, } \\
& \left.\mathbf{x}_{\text {start }, k \mid k-1}^{(j)}-\delta_{s}<\mathbf{u}<\mathbf{x}_{\mathrm{end}, k \mid k-1}^{(j)}+\delta_{e}\right\} .
\end{aligned}
$$


and the innovation covariance is given by

$$
S_{\mathrm{L}, k \mid k-1}^{(j)}=H_{a}(\mathbf{u}) P_{a, k \mid k-1}^{(j)} H_{a}(\mathbf{u})^{\mathrm{T}}+\Sigma_{a, k \mid k-1}^{(j)},
$$

where $P_{a, k \mid k-1}^{(j)}$ is the state covariance of $\mathbf{x}_{a, k \mid k-1}^{(j)}$.

Having calculated the likelihood values, two matrices of likelihood values are formed, one matrix $\Gamma_{\mathrm{P}} \in \mathbb{R}^{N_{z} \times N_{\mathrm{P}}}$ with the combinations of observations and points, according to (29), and one matrix $\Gamma_{\mathrm{L}} \in \mathbb{R}^{N_{z} \times N_{\mathrm{L}}}$ with the combinations of observations and lines, according to (31).

The association procedure using $\Gamma_{\mathrm{P}}$ and $\Gamma_{\mathrm{L}}$ must be quite unconventional because in conventional multi-target tracking, one of the well-known assumptions is that at most a single measurement can originate from a target. However, obviously, in our problem polynomials can result in multiple measurements. On the other hand, we still have the assumptions that no two measurements may originate from the same point source and no two sources may give rise to the same measurements.

Our association mechanism which uses nearest neighbor type ideas with likelihood ratio tests is summarized as follows: First find the the maximum value of $\Gamma_{\mathrm{P}}$ and call the corresponding point state $i_{\max }$ and measurement $m_{\max }$. Then, find the maximum value of the $m^{\text {th }}$ row, corresponding to measurement $m_{\max }$ of matrix $\Gamma_{\mathrm{L}}$ and call the corresponding polynomial state $j_{\max }$. The likelihood ratio denoted by $\Lambda\left(\mathbf{z}^{(m)}\right)$ is now given by

$$
\Lambda\left(\mathbf{z}^{(m)}\right) \triangleq \frac{\sqrt{\ell_{m, i_{\max }}}}{\ell_{m, j_{\max }}}
$$

where we take the square root of the point likelihood for unit matching, since the unit of (29) is (distance) $)^{-2}$ and the unit of (31) is (distance $)^{-1}$. The corresponding likelihood ratio test is

$$
\Lambda\left(\mathbf{z}^{(m)}\right) \underset{H_{1}}{\stackrel{H_{0}}{\gtrless}} \eta
$$

where $H_{0}$ and $H_{1}$ correspond to hypotheses that the measurement $\mathbf{z}^{(m)}$ is associated to the point $\mathbf{x}_{\mathbf{P}}^{\left(i_{\max }\right)}$ and to the line $\mathrm{x}_{\mathrm{L}}^{\left(j_{\max }\right)}$, respectively. The threshold $\eta$ is to be selected experimentally. More theory about likelihood ratio tests is given by e.g., [46]. When this test is performed, if the measurement $\mathbf{z}^{(m)}$ is associated to a point source $\mathrm{P}_{i}$, then the values in the $m^{\text {th }}$ row of the two matrices as well as the $i^{\text {th }}$ column of the point likelihood matrix must be set to zero to exclude the measurement and the point source from further association. However, if $\mathbf{z}^{(m)}$ is associated to line $\mathrm{L}_{j}$, then only the values in the $m^{\text {th }}$ rows of the two matrices are set to zero because the line $\mathrm{L}_{j}$ can still be associated to other measurements. The procedure is repeated until all measurements with non-zero likelihood have been associated to either a point or a line. A new point track is to be initiated if the observations could not be associated to an existing state. This is true when a measurement is not in the gate of a nonassociated point or an extended source.

\section{B. Track Handling}

Initially, our algorithm tries to generate point tracks from all incoming measurements, using any standard initialization logic. When the point tracks are established, at the end of each estimation cycle, the point track positions are examined and decisions are made on the existence of any extended sources. This process must be quite application dependent and it requires prior information on the possible forms of the polynomials to be extracted. For the purpose of giving some examples of such prior information, we go back to the five motivating application examples in Section I in the same order and list possible available prior information in each case.

- The model class of road curvatures is determined in road construction standards, and prior values may be based on the ego vehicle's motion.

- Extended objects along the road side may be based on prior knowledge of the road curvature. An example application with such details is presented in Section VI.

- The class of road inclination models are based on road construction standards and initial values may be based on maps or GPS data, to be improved in the tracking filter.

- A prior for the shore model is given by digital sea charts.

- The altitude of an island is initiated roughly based on the topographic information of a sea chart or as a standard model for islands in a given archipelago.

If some extended sources are detected in the detection process, states and covariances for this extended sources are calculated from the corresponding point tracks which are removed from the point track list after this procedure. The created extended source states and covariances, from then on, are treated as extended tracks.

When the environment changes some tracks might expire and get no measurements. When this happens, such tracks (point or extended) must be removed from the track list. For this purpose, for each track we keep a counter that holds the number of time instants that the corresponding track has not been associated to any measurement. When this counter exceeds a threshold, the corresponding track is removed from the track list.

\section{ApPlication EXAmple AND USE of PRior INFORMATION}

As an application of the ideas presented, we consider the road map estimation problem. A good road map is important for collision avoidance systems, path planing and trajectory control. Measurements from an automotive radar mounted on a moving vehicle which we refer to as the ego vehicle are used in this example which makes it possible to map the road and its surrounding infrastructure even if the line markings are bad or missing. We consider that we have the 2D world coordinate frame shown as $W$ whose origin is denoted by $O_{W}$. The state vector of the vehicle at least consists in the position and heading angle, i.e.,

$$
\mathbf{x}_{\mathrm{E}} \triangleq\left[\begin{array}{lll}
\mathrm{x}_{E W}^{W} & \mathrm{y}_{E W}^{W} & \psi_{E}
\end{array}\right]^{\mathrm{T}}
$$

with respect to the world coordinate frame $W$.

In our scenario, radar echoes typically stem from delineators or guardrails, which we would like to track as points or polynomials, respectively, with the framework presented in Section V. We use the polynomial model (4) with $n=2$ 
for our extended sources. This model has also been used for sequentially estimating similar parameters for the road's white lane markings in [47].

In the formulations of the previous sections, the extended sources are modeled as polynomials in the common $x-y$ (world) coordinates of the problem. This can be considered as a significant limitation for the applications because in real scenes the curves to be tracked might not satisfy such polynomial equations in the common $x-y$ coordinates of the problem. Here, this restriction is overcome by equipping each detected extended target with its own Cartesian $x-y$ coordinates. Each extended object with state $\mathbf{x}_{\mathrm{L}}^{(j)}$ has an associated coordinate frame denoted by $L^{(j)}$. The position of the origin and the orientation of this coordinate frame is given as

$$
\xi_{\mathrm{L}}^{(j)} \triangleq\left[\begin{array}{lll}
\mathrm{x}_{L^{(j)} W}^{W} & \mathrm{y}_{L^{(j)} W}^{W} & \psi_{L^{(j)}}
\end{array}\right]^{\mathrm{T}}
$$

with respect to the world reference frame $W$ where the superscripts are suppressed in the vector $\xi_{\mathrm{L}}^{(j)}$ for simplicity.

The measurements from the sensor mounted on the vehicle are obtained in polar coordinates (as in (2)) and need to be transformed first into the Cartesian coordinates of the sensor's frame $E_{s}$ and then into the extended source's coordinate frame in order to be used. The measurement, expressed in the extended sources coordinate frame $L$ in Cartesian coordinates is given by

$$
\mathbf{z}^{L}=\mathcal{T}^{L E_{s}}\left(\mathbf{z}^{E_{s}}, \mathbf{x}_{\mathrm{E}}, \xi_{\mathrm{L}}\right),
$$

where the transformation $\mathcal{T}^{L E_{s}}$ is described in the Appendix. Note that the measurement $\mathbf{z}^{L}$, which fits into the framework discussed in Section IV-A, is now not only affected by the original measurement $\mathbf{z}^{E_{s}}$, but also by the position of the ego vehicle, given by the state variable $\mathrm{x}_{\mathrm{E}}$, and the origin and orientation of the lanes coordinate frame $\xi_{\mathrm{L}}$. We refer to the Appendix for details about the covariance calculation for $\mathbf{z}^{L}$ involving the transformation (38).

\section{A. State Space Model}

The state space model of the extended objects was thoroughly described in Section IV and is not repeated here. The process model for the points on the form (6a) is linear and since the points are stationary, the process matrix $F_{\mathrm{P}}$ is the identity matrix. The polar measurements (2) are related to the Cartesian point states (3), i.e., $\mathbf{x}_{\mathrm{P}}=\left[\begin{array}{ll}\mathrm{x}_{P W}^{W} & \mathrm{y}_{P W}^{W}\end{array}\right]^{\mathrm{T}}$, through the measurement model according to

$$
\overline{\mathbf{z}}_{k}=\left[\begin{array}{c}
\sqrt{\left(\mathrm{x}_{P W}^{W}-\mathrm{x}_{E_{s} W}^{W}\right)^{2}+\left(\mathrm{y}_{P W}^{W}-\mathrm{y}_{E_{s} W}^{W}\right)^{2}} \\
\arctan \frac{\mathrm{y}_{P W}^{W}}{\mathrm{x}_{P W}^{W}}-\psi_{E_{s}}
\end{array}\right]+\mathbf{e}_{k},
$$

where $\left[\begin{array}{ll}\mathrm{x}_{E_{s} W}^{W} & \mathrm{y}_{E_{s} W}^{W}\end{array}\right]^{\mathrm{T}}$ and $\psi_{E_{s}}$ are the mounting position and orientation of the sensor. The measurement noise is assumed Gaussian $\mathbf{e} \sim \mathcal{N}\left(0, \Sigma_{\mathbf{p}}\right)$.

\section{B. Using Prior Information in Extended Track Generation}

An extended track is initiated from tracked points under the assumption that a number of points form a line parallel to the road. In this section we are returning to the track handling discussion started in Section V-B, in order to make the initialization procedure for extended sources more concrete. A prior information of the road's shape, or more specifically the lane's shape, in the vehicle coordinate frame is used for generating extended tracks from point tracks. The prior information is obtained by a lane tracking filter that estimates the parameters of the following lane polynomial

$$
\mathrm{y}^{E}=l_{E}+\psi_{R E} \mathrm{x}^{E}+\frac{c_{0}}{2}\left(\mathrm{x}^{E}\right)^{2},
$$

where $\mathrm{x}^{E}$ and $\mathrm{y}^{E}$ are expressed in the ego vehicle's coordinate frame $E$. The lane tracker we use is described in [47] and it is based on a ego vehicle motion model which estimates the lane given measurements from a camera, an inertia sensor and the motion of other vehicles tracked by the radar. This implies that the camera easily can be removed and that the prior solely is based on the motion model of the ego vehicle and other moving or stationary objects tracked by the radar. The parameters in (40) can be interpreted as physical sizes. The angle between the longitudinal axis of the vehicle and the road lane is $\psi_{R E}$. It is assumed that this angle is small and hence the approximation $\sin \psi_{R E} \approx \psi_{R E}$ is used. The curvature parameter is denoted by $c_{0}$ and the offset between the ego vehicle and the white lane is denoted by $l_{E}$.

All point state estimates $\hat{\mathbf{x}}_{\mathrm{P}_{i}}$ are transformed into the ego vehicles coordinate frame since the priors's geometry (40) is given in this frame. We consider hypothetical parabolas passing through each point $\hat{\mathbf{x}}_{\mathrm{P}_{k}}$ parallel to the prior (40), i.e., the parameters $\psi_{R E}$ and $c_{0}$ are just inherited from the lane tracker and the lateral distance $l_{\mathrm{P}_{k}}$ is given by

$$
l_{\mathrm{P}_{k}}=\hat{\mathrm{y}}_{\mathrm{P}_{k}}^{E}-\psi_{R E} \hat{\mathrm{x}}_{\mathrm{P}_{k}}^{E}-\frac{c_{0}}{2}\left(\hat{\mathrm{x}}_{\mathrm{P}_{k}}^{E}\right)^{2} .
$$

The likelihood $\ell_{\mathrm{P}_{i} \mathrm{P}_{k}}$ that a point $\mathrm{x}_{\mathrm{P}_{i}}$ is on the hypothetical line of point $\mathrm{P}_{k}$ is then given by

$$
\ell_{\mathrm{P}_{i} \mathrm{P}_{k}}= \begin{cases}\mathcal{N}\left(\epsilon_{\mathrm{P}_{i} \mathrm{P}_{k}} ; 0, P_{\mathrm{P}_{k},(2,2)}^{E}\right), & \text { if } \hat{\mathbf{x}}_{\mathrm{P}_{i}}^{E} \in \mathcal{G}_{\mathrm{P}_{k}} \\ 0, & \text { otherwise, }\end{cases}
$$

where the lateral distance between the point $\mathrm{P}_{i}$ and the proposed new line of point $\mathrm{P}_{k}$ is given by

$$
\epsilon_{i k}=\hat{\mathrm{y}}_{\mathrm{P}_{i}}^{E}-\hat{\mathrm{y}}_{\mathrm{P}_{k}}^{E},
$$

with

$$
\hat{\mathrm{y}}_{\mathrm{P}_{k}}^{E}=l_{\mathrm{P}_{k}}+\psi_{R E} \hat{\mathrm{x}}_{\mathrm{P}_{i}}^{E}+\frac{c_{0}}{2}\left(\hat{\mathrm{x}}_{\mathrm{P}_{i}}^{E}\right)^{2} .
$$

The notation $P_{\mathrm{P}_{k},(2,2)}^{E}$ refers to the lower-right element, i.e., the variance in the diagonal corresponding to $\mathrm{y}^{E}$. The gate $\mathcal{G}_{\mathrm{P}_{k}}$ is defined as

$$
\mathcal{G}_{\mathrm{P}_{k}} \triangleq\left\{\left[\begin{array}{c}
x \\
y
\end{array}\right] \mid \frac{\left(y-\hat{y}_{\mathrm{P}_{k}}^{E}\right)^{2}}{P_{\mathrm{P}_{k},(2,2)}^{E}} \leq \delta_{\mathrm{L}},-\delta_{s}<x-\hat{x}_{\mathrm{P}_{k}}^{E}<\delta_{e}\right\} .
$$

From all combinations of likelihoods we form a symmetric matrix $\Gamma_{\mathcal{I}}$. The columns of $\Gamma_{\mathcal{I}}$ are summed and the maximum value corresponding to column $k_{m}$ is chosen. If this column contains more than a certain number $\kappa$ of non-zero rows a parabola is formed from these points. The new line's states $\mathbf{x}_{a}$ are estimated by solving a least square problem using the corresponding points. The states $\mathbf{x}_{\text {start }}$ and $\mathbf{x}_{\text {end }}$ are the 
minimum and maximum $\mathrm{x}$-coordinate value of the points, respectively. All elements in column $k_{m}$ and rows $i_{m}$ are set to zero and the procedure is repeated until no column contains more than $\kappa$ non-zero elements.

\section{Experiments and Results}

Let us start by showing the information given by an ordinary automotive ACC radar, for the traffic situation shown in Fig. 2a. The ego vehicle, indicated by a green circle, is situated at the $(0,0)$-position in Fig. 2b, and the red dots are the radar reflections, or stationary observations, at one time sample. The smaller magenta colored dots are former radar reflections, obtained at earlier time samples. Fig. 2c shows the estimated points and lines for the same scenario using the KF EIV method presented in this contribution. The mean values of the states are indicated by solid black lines or blue points. Furthermore, the state variance, by means of the $90 \%$ confidence interval, is illustrated by gray lines or cyan colored ellipses, respectively. The estimate of the lane markings (40), illustrated by the gray dashed lines and derived according to the method presented in [47], is shown here as a comparison. We also show the tracked vehicle in front of the ego vehicle illustrated by a blue square.

In Fig. 3a we see a traffic scenario with a freeway exit. The corresponding bird's eye view is shown in Fig. 3b. The rightmost line indicates the guardrail to the right of the exit, the middle line is the guardrail starting at the exit sign. The radar measurements are indicated with red dots, the confirmed point targets with blue dots and the unconfirmed points with cyan colored dots.

Our last example shows a situation from a rural road, see Fig. $3 c$ and $3 d$. Here the road edges are not as distinct as on the freeway and the amount of clutter is higher. This leads to parallel lines with larger covariance representing the guardrail, trees etc. Appearance of many lines may already be confusing and only the mean values of the lines are plotted for sake of clarity.

These results are only snapshots from different traffic situations. To show a more rigorous result of our approach the shape of the estimated lines is compared with the recorded driven trajectory from a 3 min drive on a freeway. The recorded positions $100 \mathrm{~m}$ in front of the car are used to estimate a polynomial with parameters $a_{1}$ and $a_{2}$ at each time step $k$. It is then compared with the estimated parameters $\hat{a}_{1}$ and $\hat{a}_{2}$ of the extended objects (lines) according to the mean absolute error

$$
\text { MAE }=\frac{1}{K} \sum_{k=1}^{K}\left(\frac{1}{N_{\mathrm{L}, k}} \sum_{j=1}^{N_{\mathrm{L}, k}}\left\|\hat{a}_{1}^{(j)}-a_{1}\right\|\right)
$$

for $a_{1}$ and similarly for $a_{2}$. The polynomials are transformed to be represented in the same coordinate frame. The ego vehicle is equipped with a vision system which measures the white lanes using computer vision and represents them according to (40). The vision based parameters $\psi_{R E}$ and $c_{0} / 2$ are also compared with the trajectory as in (46) and all results are shown in Table II. Here, of course, the vision system produces better results since the driven trajectory

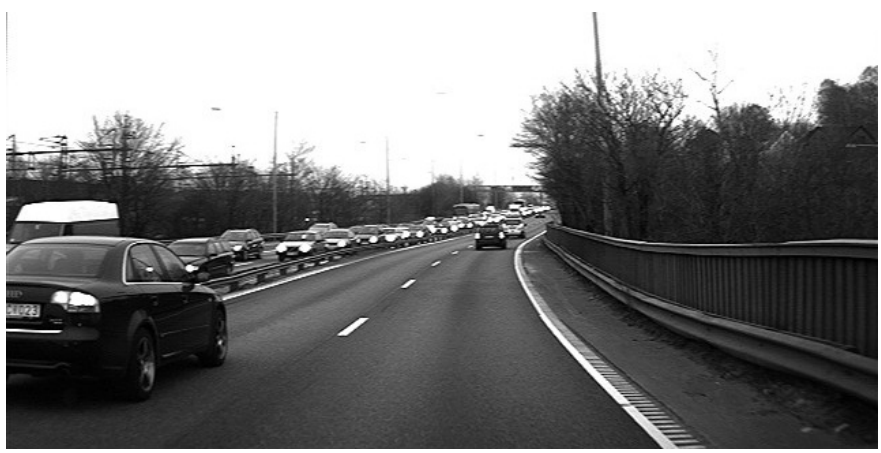

(a)

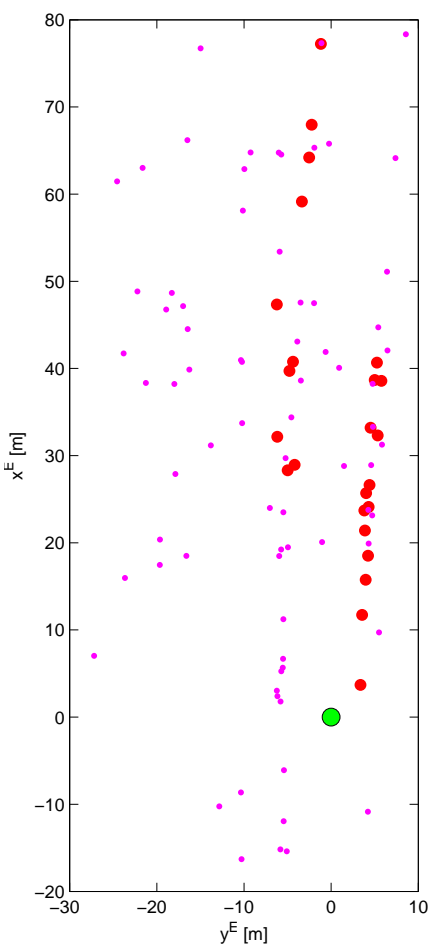

(b)

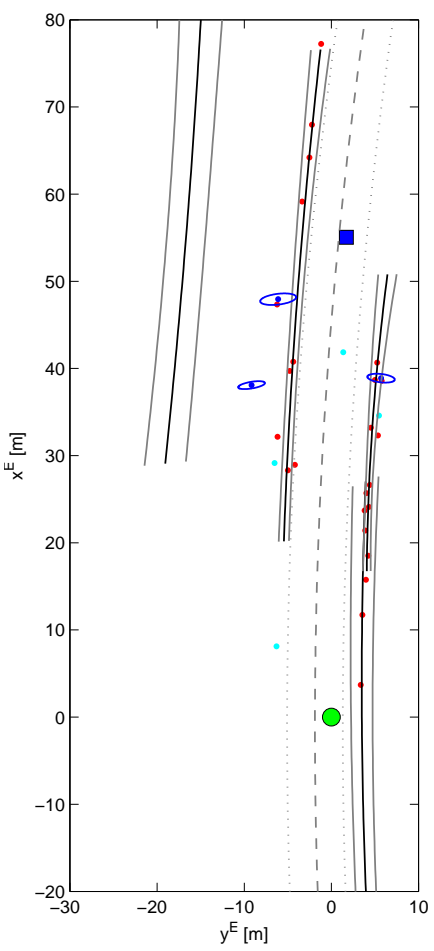

(c)
Fig. 2. A traffic situation is shown in Fig. (a). Fig. (b) shows the radar measurements, and Fig. (c) the resulting tracked points and lines. The circle in the origin is the ego vehicle, the square is the tracked vehicle in front and the dashed gray lines illustrate the tracked road curvature.

TABLE II

MAE VALUES FOR THE ESTIMATED LINES BASED ON RADAR MEASUREMENTS AND THE LANE ESTIMATE GIVEN BY THE COMPUTER VISION, BOTH WITH RESPECT TO THE DRIVEN TRAJECTORY.

\begin{tabular}{l|ll}
\hline Parameter & Radar & Vision \\
\hline$a_{1}\left(\cdot 10^{-3}\right)$ & 8.22 & 6.93 \\
$a_{2}\left(\cdot 10^{-3}\right)$ & 0.16 & 0.10 \\
\hline
\end{tabular}

should be parallel with the lane markings and the objects along the road, e.g., the guardrails, might not always be parallel. However, the table shows that our radar based approach is realistic and its performance is comparable to that of the vision based algorithm enabling the possibility of fusion with complementary information or providing estimates when other approaches fail. 


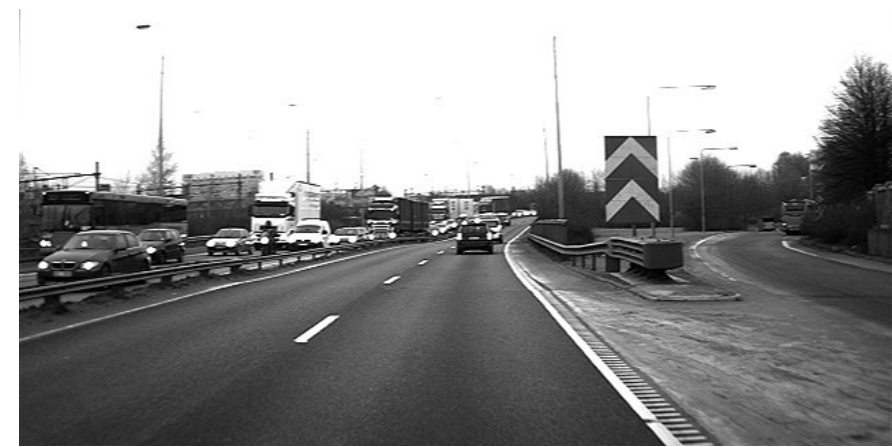

(a)

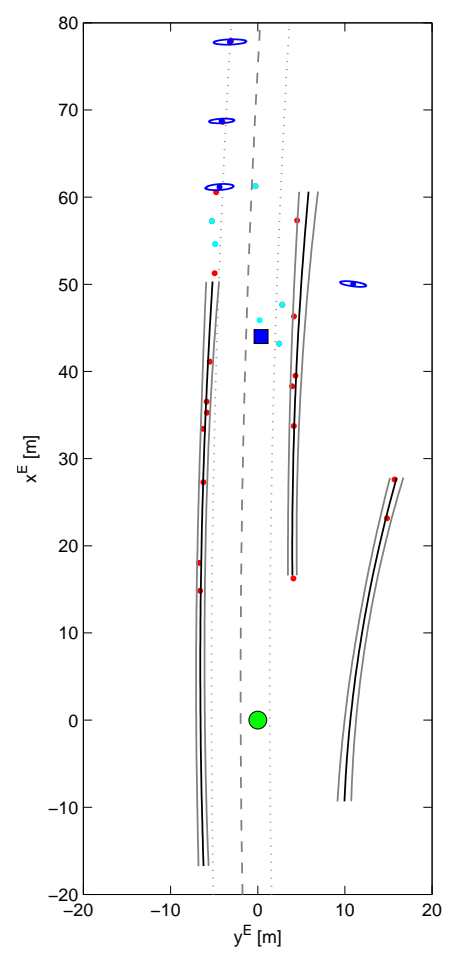

(b)

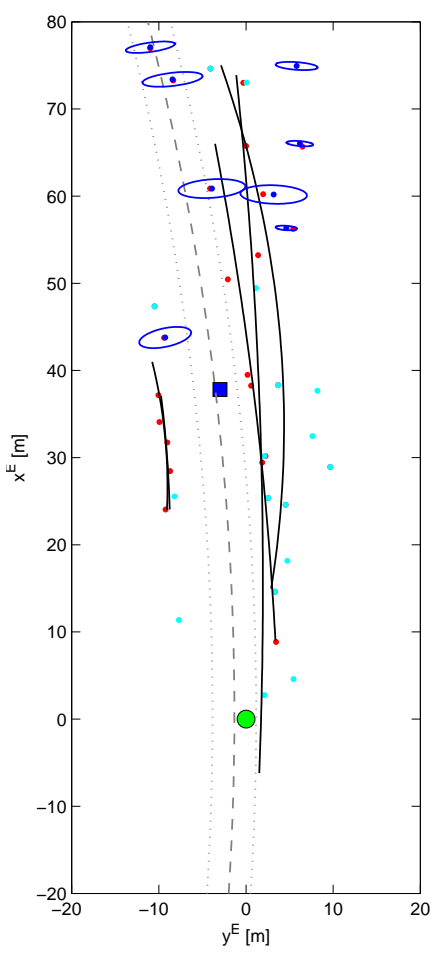

(c)

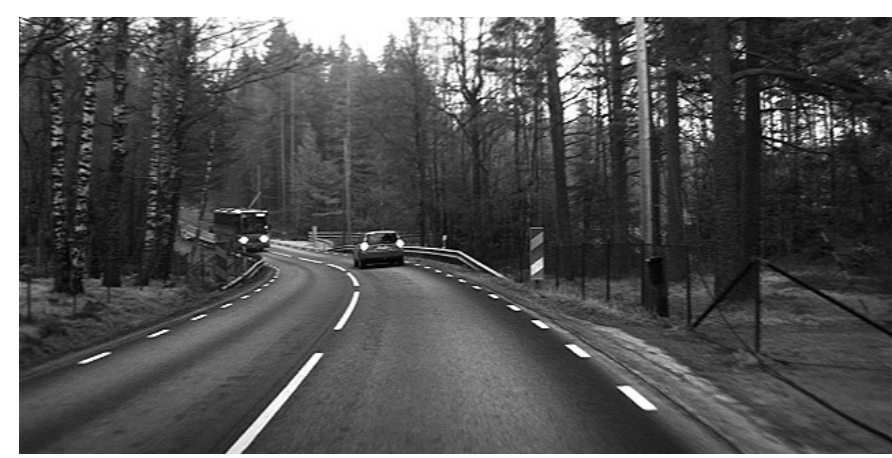

(d)

Fig. 3. Freeway exit with guardrails, the camera view is shown in Fig. (a) and the bird's eye view with the estimated states in Fig. (b). A traffic scenario from a rural road, with guardrails on both sides of a bridge is shown in Fig. (c) and (d).

\section{CONCLUSION}

In this contribution we have considered the use of polynomials in extended target tracking. State space representations that enable the use of Kalman filters for the polynomial shaped extended objects are introduced. We have also described a multi-target tracking algorithm which is as general as possible. The loss of generality of the algorithm we presented lies in that the detection and initialization of the extended tracks from point tracks would always require some application dependent prior information. In the real world application example we have presented, we tried to be as illustrative as possible about the use of this prior information.

The approach has been evaluated on real and relevant data from both freeways and rural roads in Sweden. The results are not perfect, but surprisingly good at times, and of course contain much more information than just the raw measurements. Furthermore, the standard state representation of the objects should not be underestimated since it is compact and easy to send on a vehicle CAN-bus.

Some promising future investigations include the use of non-thresholded radar data in a track-before-detect type of curve estimation and better representation of curves in terms of intrinsic coordinates.

\section{ACKNOWLEDGMENT}

The authors would like to thank the SEnsor Fusion for Safety (SEFS) project within the Intelligent Vehicle Safety Systems (IVSS) program and the strategic research center MOVIII, funded by the Swedish Foundation for Strategic Research (SSF) for financial support.

\section{APPENDIX \\ DeRIVATION OF THE CARTESIAN COVARIANCE IN THE APPLICATION EXAMPLE}

The need for a transformation from the sensor's frame $E_{s}$ into the extended source's coordinate frame $L$ was accentuated in (38) and described here in detail. The transformation $\mathcal{T}^{L E_{s}}\left(\mathbf{z}^{E_{s}}, \mathbf{x}_{\mathrm{E}}, \xi_{\mathrm{L}}\right)$ is given by

$$
\mathbf{z}^{L}=R^{L E}\left(R^{E E_{s}} \mathbf{z}^{E_{s}}+\boldsymbol{d}_{E_{s} E}^{E}\right)+R^{L W}\left(\boldsymbol{d}_{E W}^{W}-\boldsymbol{d}_{L W}^{W}\right),
$$

where the displacement vector $\boldsymbol{d}_{E_{s} E}^{E}$ is the mounting position of the sensor in the vehicles coordinate frame, the rotation matrix $R^{E E_{s}}$ describes the mounting orientation of the sensor in the vehicle and $\mathbf{z}^{E_{s}}$ is the Cartesian measurement given by the sensor.

The covariance is derived by first extracting the noise term from the stochastic variables according to

$$
\begin{aligned}
\hat{\mathbf{x}}_{\mathrm{E}} & =\mathbf{x}_{\mathrm{E}}+\tilde{\mathbf{x}}_{\mathrm{E}}, & \tilde{\mathbf{x}}_{\mathrm{E}} & \sim \mathcal{N}\left(0, P_{\mathrm{E}}\right) \\
\hat{\xi}_{\mathrm{L}} & =\xi_{\mathrm{L}}+\tilde{\xi}_{\mathrm{L}}, & \tilde{\xi}_{\mathrm{L}} & \sim \mathcal{N}\left(0, P_{\xi_{\mathrm{L}}}\right)
\end{aligned}
$$

for the ego vehicle's state variable $\mathrm{x}_{\mathrm{E}}$ and the origin of the line's coordinate frame $\xi_{\mathrm{L}}$. The noise term of the sensor measurement $\tilde{\mathbf{z}}^{E_{s}}$ was defined in (11). Equation (47) can now be rewritten according to

$$
\begin{aligned}
\mathbf{z}^{L} & =\widehat{R}^{L E}\left(R^{E E_{s}} \hat{\mathbf{z}}^{E_{s}}+\boldsymbol{d}_{E_{s} E}^{E}\right)+\widehat{R}^{L W}\left(\widehat{\boldsymbol{d}}_{E W}^{W}-\widehat{\boldsymbol{d}}_{L W}^{W}\right) \\
& +\widehat{R}^{L E} R^{E E_{s}} \tilde{\mathbf{z}}^{E_{s}}+A_{2} \tilde{\mathbf{x}}_{\mathrm{E}}+A_{3} \tilde{\xi}_{\mathrm{L}},
\end{aligned}
$$


where the rotation matrices are given by

$$
\begin{aligned}
& A_{2} \triangleq\left[\begin{array}{ccc}
\cos \hat{\psi}_{L} & \sin \hat{\psi}_{L} & -\mathrm{y}^{E_{s}} \cos \hat{\psi}_{E L}-\mathrm{x}^{E_{s}} \sin \hat{\psi}_{E L} \\
-\sin \hat{\psi}_{L} & \cos \hat{\psi}_{L} & \mathrm{x}^{E_{s}} \cos \hat{\psi}_{E L}-\mathrm{y}^{E_{s}} \sin \hat{\psi}_{E L}
\end{array}\right] \\
& A_{3} \triangleq\left[\begin{array}{ccc}
-\cos \hat{\psi}_{L} & -\sin \hat{\psi}_{L} & a_{3}^{(1,3)} \\
\sin \hat{\psi}_{L} & -\cos \hat{\psi}_{L} & a_{3}^{(2,3)}
\end{array}\right]
\end{aligned}
$$

and where

$$
\begin{aligned}
a_{3}^{(1,3)} & =\mathrm{y}^{E_{s}} \cos \hat{\psi}_{E L}+\left(\mathrm{y}_{E W}^{W}-\mathrm{y}_{L W}^{W}\right) \cos \hat{\psi}_{L} \\
& +\mathrm{x}^{E_{s}} \sin \hat{\psi}_{E L}-\left(\mathrm{x}_{E W}^{W}-\mathrm{x}_{L W}^{W}\right) \sin \hat{\psi}_{L}, \\
a_{3}^{(2,3)} & =-\mathrm{x}^{E_{s}} \cos \hat{\psi}_{E L}-\left(\mathrm{x}_{E W}^{W}-\mathrm{x}_{L W}^{W}\right) \cos \hat{\psi}_{L} \\
& +\mathrm{y}^{E_{s}} \sin \hat{\psi}_{E L}-\left(\mathrm{y}_{E W}^{W}-\mathrm{y}_{L W}^{W}\right) \sin \hat{\psi}_{L} .
\end{aligned}
$$

To summarize these calculations the covariance of $\mathbf{z}^{L}$ is given by

$$
\Sigma_{\mathrm{c}}^{L}=\widehat{R}^{L E_{s}} \Sigma_{\mathrm{c}}^{E_{s}}\left(\widehat{R}^{L E_{s}}\right)^{\mathrm{T}}+A_{2} P_{\mathrm{E}} A_{2}^{\mathrm{T}}+A_{3} P_{\xi_{\mathrm{L}}} A_{3}^{\mathrm{T}}
$$

where $\Sigma_{\mathrm{c}}^{E_{s}}$ is the Cartesian measurement covariance, $P_{\mathrm{E}}$ is the state covariance of the ego vehicles pose and $P_{\xi_{\mathrm{L}}}$ is the line state covariance corresponding to the position and orientation of the line's coordinate frame.

\section{REFERENCES}

[1] S. S. Blackman and R. Popoli, Design and Analysis of Modern Tracking Systems. Norwood, MA, USA: Artech House, 1999.

[2] Y. Bar-Shalom and X. R. Li, Multitarget-Multisensor Tracking: Principles, Techniques. Storrs, CT, USA: YBS Publishing, 1995.

[3] R. H. Bartels, J. C. Beatty, and B. A. Barsky, An introduction to splines for use in computer graphics and geometric modeling. Los Altos, CA USA: M. Kaufmann Publishers, 1987.

[4] A. Blake and M. Isard, Active contours. London, UK: Springer, 1998.

[5] F. Gustafsson, "Automotive safety systems," IEEE Signal Processing Magazine, vol. 26, no. 4, pp. 32-47, Jul. 2009.

[6] E. Hellström, "Look-ahead control of heavy vehicles," PhD thesis No 1315, Linköping Studies in Science and Technology, Linköping, Sweden, May 2010.

[7] R. Karlsson and F. Gustafsson, "Bayesian surface and underwater navigation," IEEE Transactions on Signal Processing, vol. 54, no. 11 pp. 4204-4213, Nov. 2006.

[8] H. Svensson, "Simultaneous localization and mapping in marine environment using radar images," Master's Thesis No LiTH-ISY-EX-4285, Department of Electrical Engineering, Linköping University, Sweden, 2009.

[9] T. Söderström, "Survey paper: Errors-in-variables methods in system identification," Automatica, vol. 43, no. 6, pp. 939-958, Jun. 2007.

[10] B. Ristic, S. Arulampalam, and N. Gordon, Beyond the Kalman Filter: Particle filters for tracking applications. London, UK: Artech House, 2004.

[11] M. J. Waxman and O. E. Drummond, "A bibliography of cluster (group) tracking," in Proceedings of Signal and Data Processing of Small Targets, vol. 5428. Orlando, FL, USA: SPIE, Apr. 2004, pp. 551560.

[12] R. P. S. Mahler, Statistical Multisource-Multitarget Information Fusion. Boston, MA, USA: Artech House, 2007.

[13] J. C. Dezert, "Tracking maneuvering and bending extended target in cluttered environment," in Proceedings of Signal and Data Processing of Small Targets, vol. 3373. Orlando, FL, USA: SPIE, Apr. 1998, pp. 283-294.

[14] J. Vermaak, N. Ikoma, and S. J. Godsill, "Sequential Monte Carlo framework for extended object tracking," IEE Proceedings of Radar Sonar and Navigation, vol. 152, no. 5, pp. 353-363, Oct. 2005.

[15] K. Gilholm and D. Salmond, "Spatial distribution model for tracking extended objects," IEE Proceedings of Radar, Sonar and Navigation, vol. 152 , no. 5 , pp. $364-371$, Oct. 2005
[16] K. Gilholm, S. Godsill, S. Maskell, and D. Salmond, "Poisson models for extended target and group tracking," in Proceedings of Signal and Data Processing of Small Targets, vol. 5913. San Diego, CA, USA: SPIE, Aug. 2005, pp. 230-241.

[17] Y. Boers, H. Driessen, J. Torstensson, M. Trieb, R. Karlsson, and F. Gustafsson, "Track-before-detect algorithm for tracking extended targets," IEE Proceedings of Radar, Sonar and Navigation, vol. 153, no. 4, pp. 345-351, Aug. 2006.

[18] O. E. Drummond, S. S. Blackman, and G. C. Pretrisor, "Tracking clusters and extended objects with multiple sensors," in Proceedings of Signal and Data Processing of Small Targets, vol. 1305. Orlando, FL, USA: SPIE, Jan. 1990, pp. 362-375.

[19] D. Salmond and M. Parr, "Track maintenance using measurements of target extent," IEE Proceedings of Radar, Sonar and Navigation, vol. 150, no. 6, pp. 389-395, Dec. 2003

[20] B. Ristic and D. J. Salmond, "A study of a nonlinear filtering problem for tracking an extended target," in Proceedings of the International Conference on Information Fusion, Stockholm, Sweden, Jun. 2004, pp. 503-509.

[21] D. Angelova and L. Mihaylova, "Extended object tracking using Monte Carlo methods," IEEE Transactions on Signal Processing, vol. 56, no. 2 , pp. 825-832, Feb. 2008.

[22] M. Kass, A. Witkin, and D. Terzopoulos, "Snakes: Active contour models," International Journal of Computer Vision, vol. 1, no. 4, pp. 321-331, Jan. 1988.

[23] M. Isard and A. Blake, "CONDENSATION-conditional density propagation for visual tracking," International Journal of Computer Vision, vol. 29, pp. 5-28, Aug. 1998.

[24] S. Lazarus, A. Tsourdos, B. White, P. Silson, and R. Zandbikowski, "Airborne vehicle mapping of curvilinear objects using 2-D splinegon," IEEE Transactions on Instrumentation and Measurement, vol. 59, no. 7, pp. 1941-1954, Jul. 2010.

[25] L. Ljung, System identification, Theory for the user, 2nd ed., ser. System sciences series. Upper Saddle River, NJ, USA: Prentice Hall, 1999.

[26] R. Guidorzi, R. Diversi, and U. Soverini, "Optimal errors-in-variables filtering," Automatica, vol. 39, no. 2, pp. 281-289, 2003.

[27] R. Diversi, R. Guidorzi, and U. Soverini, "Algorithms for optimal errorsin-variables filtering," Systems \& Control Letters, vol. 48, no. 1, pp. $1-13,2003$.

[28] B. Roorda and C. Heij, "Global total least squares modeling of multivariable time series," IEEE Transactions on Automatic Control, vol. 40, no. 1, pp. 50-63, Jan. 1995.

[29] R. Diversi, R. Guidorzi, and U. Soverini, "Kalman filtering in extended noise environments," IEEE Transactions on Automatic Control, vol. 50, no. 9, pp. 1396-1402, Sep. 2005.

[30] S. van Huffel and H. Zha, "An efficient Total Least Squares algorithm based on a rank-revealing two-sided orthogonal decomposition," Numerical Algorithms, vol. 4, pp. 101-133, Feb. 1993.

[31] D. L. Boley and K. Sutherland, "Recursive total least squares: An alternative to the discrete Kalman filter," in AMS Meeting on Applied Linear Algebra, DeKalb, IL, USA, May 1993.

[32] B. De Moor, "Structured total least-squares and $L_{2}$ approximationproblems," Linear algebra and its applications, vol. 188-189, pp. 163207, 1993.

[33] I. Markovsky and S. van Huffel, "Overview of total least-squares methods," Signal Processing, vol. 87, no. 10, pp. 2283-2302, 2007.

[34] Å. Björck, Numerical methods for least squares problems. Philadelphia, PA, USA: SIAM, 1996.

[35] C. Lundquist, U. Orguner, and T. B. Schön, "Tracking stationary extended objects for road mapping using radar measurements," in Proceedings of the IEEE Intelligent Vehicles Symposium, Xi'an, China, Jun. 2009, pp. 405-410.

[36] A. Kirchner and T. Heinrich, "Model based detection of road boundaries with a laser scanner," in Proceedings of the IEEE Intelligent Vehicles Symposium, Stuttgart, Germany, Oct. 1998, pp. 93-98.

[37] A. Polychronopoulos, A. Amditis, N. Floudas, and H. Lind, "Integrated object and road border tracking using $77 \mathrm{GHz}$ automotive radars," IEE Proceedings of Radar, Sonar and Navigation, vol. 151, pp. 375-381, Dec. 2004.

[38] C. Lundquist and T. B. Schön, "Estimation of the free space in front of a moving vehicle," in Proceedings of the SAE World Congress, ser. SAE paper 2009-01-1288, Detroit, MI, USA, Apr. 2009.

[39] W. Wijesoma, K. Kodagoda, and A. Balasuriya, "Road-boundary detection and tracking using ladar sensing," IEEE Transactions on Robotics and Automation, vol. 20, no. 3, pp. 456-464, Jun. 2004. 
[40] K. R. S. Kodagoda, W. S. Wijesoma, and A. P. Balasuriya, "CuTE: curb tracking and estimation," IEEE Transactions on Control Systems Technology, vol. 14, no. 5, pp. 951-957, Sep. 2006.

[41] B. Fardi, U. Scheunert, H. Cramer, and G. Wanielik, "Multi-modal detection and parameter-based tracking of road borders with a laser scanner," in Proceedings of the IEEE Intelligent Vehicles Symposium, Columbus, OH, USA, Jun. 2003, pp. 95-99.

[42] Y. Wang, L. Bai, and M. Fairhurst, "Robust road modeling and tracking using condensation," IEEE Transactions on Intelligent Transportation Systems, vol. 9, no. 4, pp. 570-579, Dec. 2008.

[43] P. J. Huber and E. Ronchetti, Robust Statistics. New York, NY, USA: John Wiley \& Sons, 2009.

[44] Y. Bar-Shalom and T. E. Fortmann, Tracking and Data Association, ser. Mathematics in science and engineering. Orlando, FL, USA: Academic Press, 1988.

[45] D. B. Reid, "An algorithm for tracking multiple targets," IEEE Transactions on Automatic Control, vol. 24, no. 6, pp. 843-854, 1979.

[46] H. L. van Trees, Detection, Estimation, and Modulation Theory. New York, NY, USA: John Wiley \& Sons, 1968.

[47] C. Lundquist and T. B. Schön, "Joint ego-motion and road geometry estimation," Information Fusion, 2010, DOI: 10.1016/j.inffus.2010.06.007.

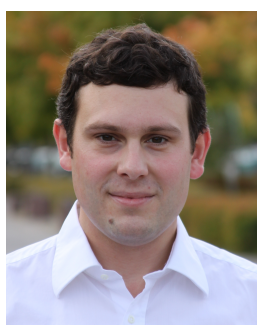

Christian Lundquist is a $\mathrm{PhD}$ student at the $\mathrm{Di}$ vision of Automatic Control at the Department of Electrical Engineering of Linköping University. Between 2004 and 2007 he worked with active steering systems at the German company ZF Lenksysteme $\mathrm{GmbH}$. In 2003 he received the M.Sc. degree in Automation and Mechatronics Engineering from Chalmers University of Technology in Gothenburg, Sweden. His research interests include sensor fusion and target tracking for automotive applications.

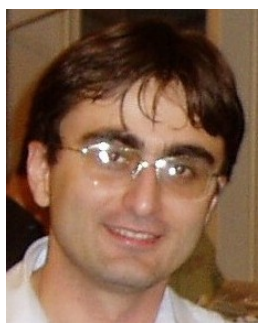

Umut Orguner (M'00) is an Assistant Professor at the Division of Automatic Control at the Department of Electrical Engineering of Linköping University. He held a postdoctoral position at the same department between 2007 and 2009. He got his B.Sc., M.Sc. and Ph.D. degrees all in Electrical Engineering from Department of Electrical and Electronics Engineering, Middle East Technical University, Ankara, Turkey in 1999, 2002 and 2006 respectively. His research interests include estimation theory, multiple-model estimation, target tracking,

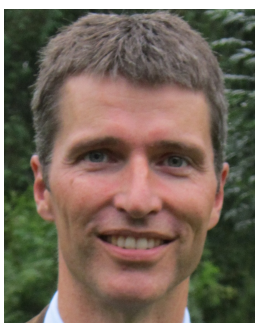

Fredrik Gustafsson (S'91-M'93-SM'05) is professor in Sensor Informatics at Department of Electrical Engineering, Linköping University, since 2005. He received the M.Sc. degree in electrical engineering 1988 and the Ph.D. degree in Automatic Control, 1992, both from Linköping University. During 19921999 he held various positions in automatic control, and 1999-2005 he had a professorship in Communication Systems. His research interests are in stochastic signal processing, adaptive filtering and change detection, with applications to communication, vehicular, airborne, and audio systems. His work in the sensor fusion area involves design and implementation of nonlinear filtering algorithms for localization, navigation and tracking of all kind of platforms, including cars, aircraft, spacecraft, UAV's, surface and underwater vessels, cell phones and film cameras for augmented reality. He is a co-founder of the companies NIRA Dynamics and Softube, developing signal processing software solutions for automotive and music industry, respectively.

He was an associate editor for IEEE Transactions of Signal ProCESSING 2000-2006 and is currently associate editor for EURASIP Journal on Applied Signal Processing and International Journal of Navigation and Observation. In 2004, he was awarded the Arnberg prize by the Royal Swedish Academy of Science (KVA) and in 2007 he was elected member of the Royal Academy of Engineering Sciences (IVA). 